\title{
NOUVELle
}

\section{Le protéasome limite la transcription dans les cellules souches embryonnaires}

Henrietta Szutorisz, Niall Dillon, Laszlo Tora
L. Tora : Institut de Génétique et de Biologie Moléculaire

et Cellulaire (IGBMC), CNRS UMR7104, Inserm U596,

Université Louis Pasteur, 67400 IIIkirch, France.

laszlo@igbmc.u-strasbg.fr

H. Szutorisz: Stowers Institute for Medical Research, 1000 East 50th Street, Kansas City, M0 64110, États-Unis. hsz@stowers-institute.org

N. Dillon : Gene Regulation and Chromatin Group,

MRC Clinical Sciences Centre,

Faculty of Medicine, Imperial College,

Hammersmith Hospital Campus,

Du Cane Road, London W12 ONN,

Royaume-Uni.

niall.dillon@csc.mrc.ac.uk

les eucaryotes supérieurs. Les cellules souches embryonnaires (ES) ont la capacité d'activer tous les programmes d'expression de gènes somatiques pendant leur différenciation, et, par conséquent, sont capables de produire tous les types de cellules différenciées de l'organisme. Cette propriété, nommée pluripotence, soulève des questions importantes sur la façon dont la compétence transcriptionnelle des gènes spécifiques de tissus est maintenue dans les cellules ES. Plusieurs études montrent que, dans les cellules $\varepsilon S$, des gènes spécifiques de tissus sont déjà « préparés » alors même que leur transcription ne se déroulera que plus tard au cours du développement. Cet état de compétence, où les gènes sont «préparés» mais ne sont pas encore exprimés, est important pour maintenir la pluripotence des cellules $\varepsilon S$ et empêcher leur différenciation [1, 2]. De plus, la chromatine des cellules $E S$ est généralement considérée comme hyperdynamique [3]. Dans les cellules $\varepsilon S$, certaines régions du génome contiennent des modifications dites « actives» des histones qui forment des marques précoces de compétence transcriptionnelle $[4,5]$. Il a été montré que ces marques épigénétiques constituent des sites de recrutement de facteurs de transcription alors même que ces gènes ne sont pas encore transcrits [4, 5] (Figure 1).

Les modifications de la chromatine, le recrutement des facteurs généraux de transcription (GTF) comme TFIIA-H et l'assemblage du complexe de pré-initiation (PIC) à partir des GTF sont les points de régulation principaux de la transcription, parce qu'ils indiquent où et quand la transcription par l'ARN polymérase II (Pol II) sera initiée [6, 7]. Dans les cellules ES, caractérisées par un environnement chromatinien permissif, le contrôle de la formation du PIC est donc encore plus crucial que dans des cellules différenciées. De plus, l'environnement permissif de la chromatine dans des cellules ES suggère l'existence de mécanismes limitant la liaison non spécifique des facteurs de transcription.

Le protéasome a été récemment impliqué dans le contrôle dynamique de la liaison des facteurs de transcription aux promoteurs des gènes actifs [8]. Le protéasome 265 est un complexe multiprotéique très conservé exerçant une activité protéasique et jouant un rôle central dans le contrôle de la stabilité des protéines dans les cellules eucaryotes. Le complexe 26S est organisé en deux sous-complexes, le noyau protéolytique $20 \mathrm{~S}$ et la particule régulatrice 19S. Le complexe 19S est luimême fait de deux composants, la base et le couvercle. La base contient des ATPases et des sous-unités non enzymatiques, et le couvercle les protéines qui semblent être responsables de la liaison spécifique des substrats [9].

Bien que leur chromatine soit largement ouverte et considérée comme permissive, les cellules ES ne permettent pas la transcription d'une variété de gènes codant pour des facteurs de différenciation cellulaire, ni l'initiation cryptique à partir de régions intergéniques. Ces observations nous ont incité à formuler l'hypothèse selon laquelle le protéasome pourrait contrôler, dans les cellules $\varepsilon S$, les processus de transcription non désirés de régions intergéniques ou de gènes de différenciation [10]. Pour évaluer cette hypothèse, nous avons sélectionné le locus $\lambda 5$ VpréBl ${ }^{l}$, qui n'est pas actif dans les cellules $E S$, mais est activé pendant les phases précoces de différenciation des progéniteurs des lymphocytes B. Quand des inhibiteurs chimiques du protéasome, ou des ARN interférents inhibant l'expression des sous-unités du protéasome, sont ajoutés aux cellules \&S, nous avons observé une augmentation de la transcription de certaines régions intergéniques dans le locus $\lambda 5$-VpréBl dans ces cellules. Nous avons également observé que l'inhibition du protéasome dans les cellules \&S déclenche l'apparition de nouveaux sites d'initiation de la transcription [10]. Cet effet n'est pas vu dans des cellules diffé-

\footnotetext{
${ }^{1}$ Chez la souris, ce gène contrôle l'expression d'une protéine qui s'associe à la chaîne lourde $\mu$ des immunoglobulines, intracytoplasmique, et permet l'expression du pré-BCR à la surface des précurseurs $B$. En son absence, la différenciation est bloquée.
} 
renciées, suggérant qu'il est spécifique aux cellules $\varepsilon S$.

Pour explorer plus en détail le rôle du protéasome dans la transcription, nous avons examiné si l'inhibition de son activité aurait un effet direct sur la liaison du PIC ou des facteurs de remodelage de la chromatine au locus $\lambda 5$ VpreBl, et plus particulièrement aux régions intergéniques dont la transcrip- tion est anormale lorsque le protéasome est inhibé. En effet, nous avons constaté que les facteurs comme TBP (sous-unité du complexe TFIID qui fait partie du PIC), Brgl (brahma-related gene 1, sousunité du complexe de remodelage de la chromatine SWI/SNF), TRRAP (transformation/transcription domain-associated protein, sous-unité des complexes de remodelage de la chromatine STAGA/

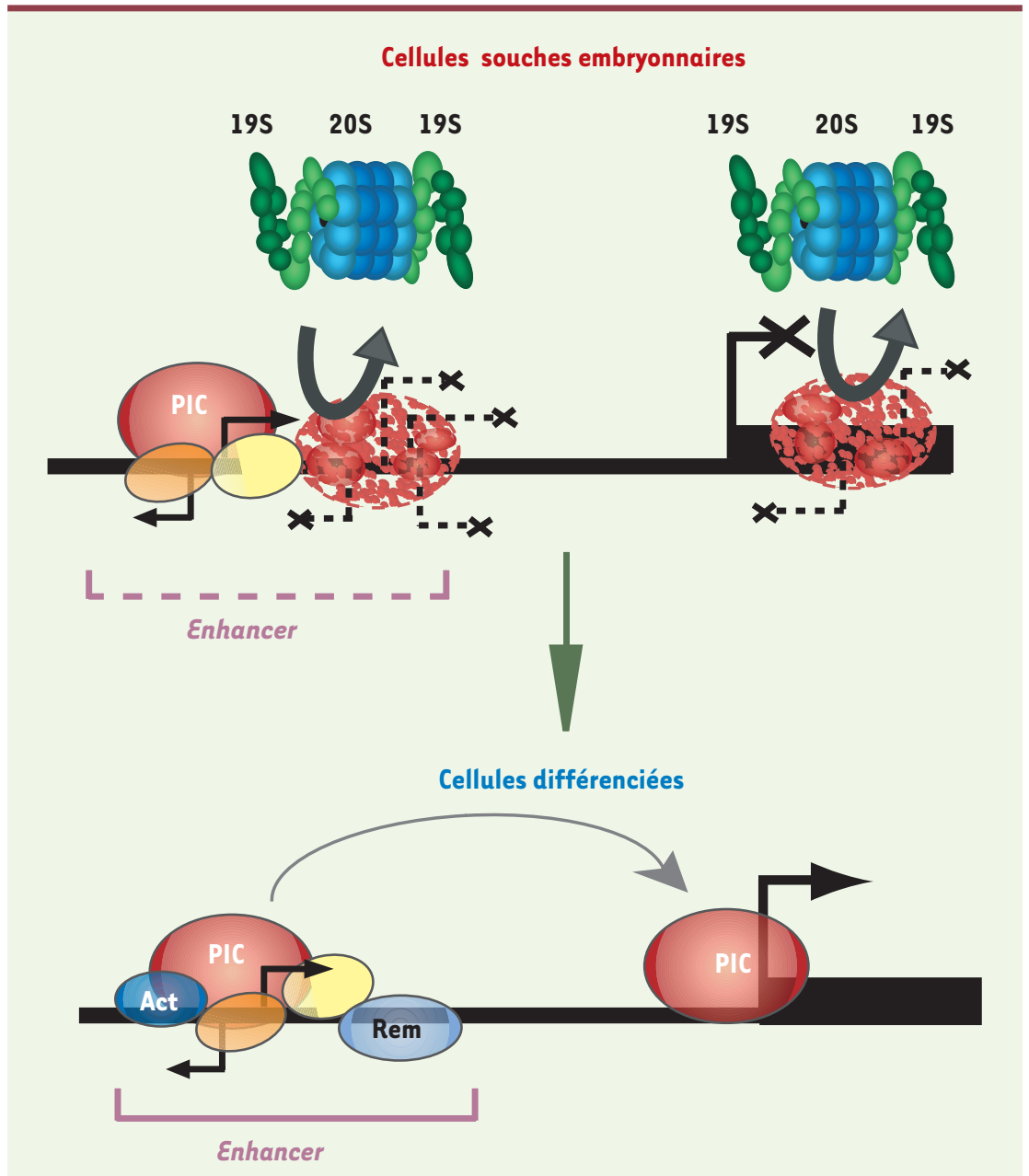

Figure 1. Modèle pour le contrôle de la transcription par le protéasome dans les cellules ES. Les gènes spécifiques de tissus contiennent des enhancers qui agissent comme centres de recrutement des facteurs de transcription [5]. Dans des cellules ES, le protéasome 26S (composé du noyau protéolytique $20 S$ et du domaine régulateur 195 ) cible les PIC non-spécifiques (rouge dégradé) assemblés sur des promoteurs cryptiques, pour empêcher la transcription inter- et intragénique (lignes pointillées). La conformation et/ou la composition anormale de ces PIC en feraient des cibles pour la dégradation par le protéasome. Dans les cellules différenciées, le recrutement des activateurs (Act) spécifiques de tissu au niveau des enhancers permet le remodelage de la chromatine par le recrutement des complexes de remodelage de la chromatine (Rem) et l'assemblage des PIC stables et normaux (rouge) sur les promoteurs des gènes spécifiques de tissus. Ceux-ci ne sont plus sujets à la dégradation par le protéasome.

TFTC ou NuA4/TIP60), ainsi que la Pol II n'étaient liés à ces régions qu'après l'inhibition du protéasome. Nous avons ensuite démontré que les sous-unités du protéasome, aussi bien celles du $20 S$ et du 19S, étaient liées au locus $\lambda 5$-VpréB 1 dans des conditions normales. De plus, nous avons constaté que ce processus était très dynamique et dépendant de l'activité du protéasome [10].

Nous avons également analysé, dans des cellules ES, les effets de l'inhibition du protéasome sur deux locus additionnels, ceux des gènes de la $\beta$-globine et de HoxD4, dont l'expression est régulée pendant le développement. Comme c'était le cas pour le locus $\lambda 5$-VpréBl, I'inhibition du protéasome entraîne également une augmentation de la transcription de ces deux locus et de la liaison des facteurs de transcription, suggérant que ce mécanisme s'applique peut-être à beaucoup d'autres régions génomiques des cellules \&S [10].

De plus, nos données indiquent que les activités des promoteurs sont sensiblement plus fortes dans les cellules $\varepsilon S$ que cela n'avait été précédemment décrit. Mais cette transcription est continuellement réprimée par le protéasome qui enlève les PIC assemblés sur des promoteurs cryptiques (Figure 1). Nous faisons I'hypothèse que, dans des régions interou intragéniques des cellules ES, les PIC ont une conformation et/ou une composition modifiées par rapport à celles des PIC normaux; ces derniers peuvent être stabilisés par des activateurs spécifiques dans des cellules différenciées (Figure 1), alors que la structure anormale et nonspécifique de ces PIC dans les cellules indifférenciées de type ES en ferait des cibles pour la dégradation par le protéasome [11].

La répression de la transcription qu'exerce de façon non spécifique le protéasome ajoute une nouvelle dimension au contrôle de la pluripotence des cellules ES et identifie une fonction entièrement nouvelle pour le protéasome. Nos résultats suggèrent que les régions génomiques contenant des gènes spécifiques de lignées 
de différenciation sont dans un état fortement dynamique dans les cellules ES: des facteurs se lient en permanence aux séquences régulatrices, mais sont enlevés par le protéasome. Ce contrôle est susceptible de maintenir les gènes spécifiques de tissus dans un état transcriptionnellement compétent, sans pour cela qu'ils soient transcrits, permettant ainsi leur activation rapide et efficace lors de la différenciation cellulaire. $\diamond$

Suppression of non-specific transcription by the proteasome in embryonic stem cells

\section{RÉFÉRENCES}

1. Boyer LA, Lee TI, Cole MF, et al. Core transcriptional regulatory circuitry in human embryonic stem cells. Cell $2005 ; 122$ : 947-56.

2. Szutorisz H, Dillon $N$. The epigenetic basis for embryonic stem cell pluripotency. Bioessays 2005 ; 27 : 1286-93.

3. Meshorer $\varepsilon$, Yellajoshula D, George $\varepsilon$, et al. Hyperdynamic plasticity of chromatin proteins in pluripotent embryonic stem cells. Dev Cell 2006 ; $10: 105-16$.

4. Szutorisz H, Canzonetta C, Georgiou A, et al. Formation of an active tissue-specific chromatin domain initiated by epigenetic marking at the embryonic stem cell stage. Mol Cell Biol 2005 ; 25 : 1804-20.

5. Szutorisz H, Dillon N, Tora L. The role of enhancers as centres for general transcription factor recruitment. Trends Biochem Sci $2005 ; 30$ : 593-9.

\section{NOUVELLE}

\section{Nouveaux mécanismes régulateurs de Bcl10 Une avancée dans la compréhension de la survenue des lymphomes du MALT ? \\ Camille Lobry, Robert Weil}

6. Martinez $\varepsilon$. Multi-protein complexes in eukaryotic gene transcription. Plant Mol Biol 2002 ; 50 : 925-47.

7. Muller F, Tora L. The multicoloured world of promoter recognition complexes. EMBO J $2004 ; 23: 2-8$.

8. Collins GA, Tansey WP. The proteasome: a utility tool for transcription? Curr Opin Genet Dev 2006 ; $16: 197-202$

9. Groll M, Bochtler M, Brandstetter H, et al. Molecular machines for protein degradation. Chembiochem $2005 ; 6: 222-56$

10. Szutorisz H, Georgiou A, Tora L, et al. The proteasome restricts permissive transcription at tissue-specific gene loci in embryonic stem cells. Cell 2006 ; $127: 1375-88$.

11. Asher G, Reuven N, Shaul Y. 20 S proteasomes and protein degradation "by default". Bioessays 2006; $28: 844-9$.
Unité de Signalisation Moléculaire

et Activation Cellulaire,

URA 2582 Centre National de la Recherche Scientifique (CNRS), Institut Pasteur,

25 , rue du Docteur Roux,

75724 Paris Cedex 15, France.

rweil@pasteur.fr

\section{> NF-KB et lymphomes du MALT}

La voie de signalisation NF- $\kappa$ B (Figure 1) joue un rôle important dans les réponses immune, inflammatoire et anti-apoptotique, à la fois au cours des processus de différenciation (en particulier dans le système hématopoiétique) et de cancérogenèse [1]. Dans ce dernier cas, son implication est liée en grande partie à la présence, parmi ses gènes cibles, d'un grand nombre de gènes de résistance à l'apoptose, tels que $B c l-X L, X I A P, A l$, $I \varepsilon X-1 L$ et $G A D D 45 \beta$ ainsi que de gènes impliqués dans la croissance tumorale, comme la cycline DI et le proto-oncogène $c-m y c$.

Les lymphomes du MALT (mucosa-associated lymphoid tissue) sont un exemple de cancers impliquant NF- $\kappa B$. Ce sont des formes hétérogènes de lymphomes non hodgkiniens impliquant une prolifération lymphoïde B dont l'origine est la zone marginale des follicules lymphoïdes associés aux muqueuses. Les lymphomes du MALT sont souvent associés à une stimulation antigénique chronique impliquant les pathogènes suivants: H. pylori dans le lymphome du MALT gastrique, C. jejuni dans le lymphome du MALT intestinal, B. bugdorferi dans le lymphome du MALT cutané, et enfin très récemment C. psittaci dans le lymphome du MALT oculaire. Quatre translocations chromosomiques, $\mathrm{t}(11 ; 18)(\mathrm{p} 21 ; \mathrm{q} 21)$, $\mathrm{t}(1 ; 14)(\mathrm{p} 22 \mathrm{q} 32), \mathrm{t}(14 ; 18)(\mathrm{q} 32 ; \mathrm{q} 21)$ et $\mathrm{t}(3 ; 14)(\mathrm{pl3} ; \mathrm{q} 32)$ sont spécifiquement associées aux lymphomes du MALT. Les translocations $\mathrm{t}(1 ; 14)(\mathrm{p} 22 \mathrm{q} 32)$ et $t(14 ; 18)(q 32 ; q 21)$ fusionnent respectivement le gène $B C L 10$ et le gène MALTI avec le promoteur des chaînes lourdes des immunoglobulines tandis que $\mathrm{t}(11 ; 18)(\mathrm{p} 21 ; \mathrm{q} 21)$ fusionne les gènes $A P I 2$ et MALT1. Les produits des gènes MALTI et $B C L 10$ sont directement impliqués dans les voies de signalisation menant à l'activation de NF- $\kappa B$ par le récepteur des lymphocytes $B(B C R)$ et le récepteur des lymphocytes T (TCR).
Des effecteurs communs aux voies du BCR ( $B$ cell receptor) et du TCR (T cell receptor) ont été identifiés [2] dont: (1) CARMAl, qui fait partie d'une famille caractérisée par la présence d'un domaine CARD (caspase recruitement domain) amino-terminal, d'un domaine coiled-coil et d'un motif carboxy-terminal «MAGUK» (membrane-associated guanylate kinase) contenant des motifs PDZ, SH3 et guanylate (GUK); (2) Bcllo, une protéine possédant un domaine homotypique CARD et un domaine riche en sérine et thréonine; et (3) la paracaspase MALTI.

Différentes approches biochimiques et génétiques ont permis de déterminer l'ordre chronologique des événements conduisant à l'activation de NF- $\kappa B$ par le récepteur des lymphocytes $T$ : dans un premier temps, CARMAl est phosphorylée par $\mathrm{PKC} \theta$, ce qui permet le recrutement de Bcl10/MALTI pour former le complexe CARMAl/Bcl10/MALTl (CBM). Dans un deuxième temps, le complexe NEMO/IKK 